\title{
A memory clinic $v$. traditional community mental health team service: comparison of costs and quality
}

\author{
Judy Sasha Rubinsztein, ${ }^{1}$ Marelna Janse van Rensburg, ${ }^{2}$ Zerak Al-Salihy, ${ }^{1}$ Deborah Girling, ${ }^{2}$ \\ Louise Lafortune, ${ }^{3}$ Muralikrishnan Radhakrishnan, ${ }^{4}$ Carol Brayne ${ }^{3}$
}

BJPsych Bulletin (2015), 39, 6-11, doi: 10.1192/pb.bp.113.044263

\author{
${ }^{1}$ Norfolk and Suffolk NHS Foundation \\ Trust; ${ }^{2}$ Cambridgeshire and \\ Peterborough NHS Foundation Trust; \\ ${ }^{3}$ University of Cambridge; ${ }^{4}$ King's \\ College London \\ Correspondence to Judy Rubinsztein \\ (judy.rubinsztein@nsft.nhs.uk) \\ First received 27 May 2013, final \\ revision 5 Sep 2013, accepted 30 Oct \\ 2013 \\ (C) 2014 The Royal College of \\ Psychiatrists. This is an open-access \\ article published by the Royal College \\ of Psychiatrists and distributed \\ under the terms of the Creative \\ Commons Attribution License (http:// \\ creativecommons.org/licenses/by/ \\ 4.0), which permits unrestricted use, \\ distribution, and reproduction in any \\ medium, provided the original work \\ is properly cited.
}

\begin{abstract}
Aims and method To compare the cost and quality of a memory-clinic-based service (MCS) with a traditional community mental health team (CMHT) service. Using a retrospective case-note review, we studied two groups, each with 33 participants. Consecutive referrals for diagnostic 'memory' assessments over 4 months were evaluated. Participants were evaluated for up to 6 months.
\end{abstract}

Results The MCS was less costly than the CMHT service but the difference was not statistically significant (mean cost for MCS was $£ 742$, mean cost for CMHT service

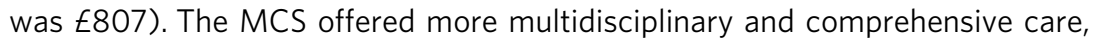
including: pre- and post-diagnostic counselling, more systematic screening of blood for reversible causes of dementia, more use of structured assessment instruments in patients/carers, signposting to the third sector as well as more consistent copying of letters to patients/carers.

Clinical implications An MCS service offered more comprehensive and multidisciplinary service at no extra cost to secondary care.

Declaration of interest None.
In the UK, the national dementia strategy ${ }^{1}$ strongly supports the concept of timely diagnosis and intervention in dementia. The cost-effectiveness of such services has been demonstrated. ${ }^{2-6}$ Studies show that memory services may improve quality of life for patients and carers. ${ }^{7-9}$ The recognition of dementia enables the provision of safer care by considering, for example, accommodation and care needs and encouraging advanced decision-making. Reaching a diagnosis can help patients and carers to tolerate their symptoms better and suitable treatments can be considered.

Advocates of dedicated 'memory clinic services' (MCSs) point to the benefits of a less stigmatising setting, with a focus on psychosocial interventions, education and the promotion of research and clinical governance. .,7,10-14 $^{-1}$ Opponents are concerned with resources being diverted from community mental health teams (CMHTs) and point to higher costs of MCS services. ${ }^{15,16}$

In this study, Trust A has a dedicated memory clinic service (henceforth called MCS group) as well as a CMHT. In Trust B there is a 'traditional' CMHT-based service (henceforth called CMHT group) where referrals of all types are seen. We compared the costs with those of secondary care and the quality of the service offered to similar types of patients referred for a non-urgent diagnostic memory assessment in these two service models in neighbouring trusts. Patients were evaluated retrospectively using casenote review in terms of the cost and quality of care they received in secondary care for up to 6 months in similar rural/suburban areas.

\section{Method}

Study design

This study was a health service evaluation and used retrospective data collected routinely as part of the usual services provided in both trusts, so local research ethics committee permission was not needed.

\section{Demographic matching}

Two rural and suburban eastern areas of England were matched using data from the Eastern Region Public Health Observatory (ERPHO). The number of people over the age of 65 years, based on general practice (GP) records in these areas, was recorded: 20289 people in the MCS group and 21112 people in the CMHT group. Data from the British primary care Quality and Outcomes Framework (QOF), ${ }^{17}$ which requires the recording of dementia and other chronic 
conditions, showed that the prevalence of dementia and strokes, and the recording of cholesterol and blood pressure, were similar in both groups. The Indices of Multiple Deprivation (IMD) ${ }^{18}$ show that the GP practices in the CMHT service (Trust B) experienced less deprivation. The range of the IMD was 11.5-17.7 for the MCS and 5.7-8.96 for the CMHT service.

\section{Patient identification}

We examined all referrals received over the time frame of the study (4 months) from specific, predetermined GP practices in these two rural/suburban areas. All patients included in the study had to fulfil Department of Health memory clinic referral criteria. ${ }^{19}$ These criteria state that memory services will see all patients with subjective memory problems or change in everyday function, or a carer's report of change in a patient over a period of more than 6 months. Patients referred should have no previous or definitive diagnosis of dementia. The Department of Health stipulates that memory services will not see patients where urgent treatment is needed, for example those with more complex behavioural and psychological problems, patients with suicidal ideation, psychotic behaviour or a crisis situation from the carer's perspective. Patients with earlyonset dementia (age under 64 years) were also excluded because the two areas have different referral pathways for such patients. The MCS service received two referrals of early-onset dementia, which were excluded from this study; there were no such referrals in the CMHT service. All referrals had to have been received between August and November 2011 in both services.

For the MCS group, a computerised search of all memory clinic patients referred by GP practices in the designated area of the trust was performed for the specified time period. Thirty-four consecutive patients were identified and all fulfilled the Department of Health memory clinic criteria. ${ }^{19}$ One patient refused the diagnostic interview after pre-diagnostic counselling and that patient was excluded. Occasionally, patients may be passed on to the crisis team or CMHT, but none on our list had been dealt with in this way.

For the CMHT group, a member of the research team (M.J.V.R.) manually searched all consecutive referrals to the service from particular GP practices from a central written database. Thirty-three referrals were included in the CMHT group (by coincidence, the same number of patients as the MCS group). These 33 patients all fulfilled Department of Health memory clinic criteria $^{19}$ and were identified by consecutively recruiting from the original list. Patients were excluded only if they did not fulfil memory clinic criteria. ${ }^{19}$ A second member of the research team (J.S.R.) ensured that all referrals met these criteria for inclusion or exclusion. There was uncertainty as to whether to include one patient and a senior nurse's opinion from the MCS team was sought to arbitrate.

\section{Questionnaires}

Quality of service

A data extraction sheet to assess the quality of the service was developed for this study. It was used for case-note analysis and captured information routinely collected by clinicians for assessment purposes (Box 1). The quality criteria were chosen based on the National Institute for Health and Care Excellence (NICE) guidance for dementia services, ${ }^{20}$ the Memory Services National Accreditation Programme (MSNAP) criteria where they could be applied to both services, ${ }^{21}$ and literature evidence. ${ }^{10,11,22}$ We pragmatically assessed whether the data could be extracted retrospectively from the services. This information was obtained from computerised and handwritten notes from the initial contact with the patient and for the following 6 months of contact with the mental health service. The psychiatrists involved in the data collection conferred about any uncertainties with recording of data. M.J.V.R. and Z.A.-S. each initially screened at least four sets of case records together with J.S.R. to ensure interrater reliability in recording of data. All entries were scrutinised by J.S.R. to ensure data entry was consistent. Any missing information was noted. It is the view of clinicians in both trusts that GPs are best suited to conduct physical examinations and these are stated in the requirements for referral to the service by the MCS trust. However, individual psychiatrists often choose to conduct some aspects of the physical examination themselves and the extent to which clinicians were doing this was noted.

\section{Cost of service}

The Client Service Receipt Inventory (CSRI) ${ }^{23}$ was adapted for this study (available from the authors on request). This includes all the mental health service costs for each individual patient from the initial point of contact and then all subsequent contacts with the mental health service

Box 1 Measures extracted regarding the quality of the memory service

- Background characteristics (age, gender, marital status, employment, school-leaving age, accommodation)

- Waiting time to be seen

- Symptom time prior to referral

- Presence of a carer, relative, friend

- Pre-diagnostic counselling

- Dementia blood screen: ordered, examined

- Informal assessment of functioning, behaviour, depression, global assessment

- Structured questionnaires to assess functioning, behaviour global assessment

- Brain imaging

- Physical examination (record from GP/psychiatrist)

- MMSE, ACE-R or other cognitive tools utilised

- Record of a diagnosis

- Record of risk assessment

- Record of post-diagnostic advice to patient/carer

- Record of discussion about driving

- Copying of letters to patients/carers

ACE-R, Addenbrooke's Cognitive Examination-Revised; GP, general practitioner; MMSE, mini-Mental State Examination. 
over the following 6 months. Costing stopped at the point the patient was discharged back to the GP. However, patients found to need more extensive follow-up for more severe or complex behavioural and psychological symptoms of dementia (BPSD) were referred to the CMHT service in Trust A (one patient) and Trust B (three patients). Costs were excluded from that point. Costing was calculated from the perspective of National Health Service care ${ }^{24}$ (secondary care only evaluated in this study) rather than from a wider medical or societal perspective. Costs for hourly contact with professionals were mainly derived from the unit costs for health and Social Services compendium and included 'on costs', for example for a consultant psychiatrist this includes salary, national insurance and superannuation, qualifications, overheads, ongoing training and capital overheads. ${ }^{25}$ From this document, ${ }^{25}$ the cost per hour for consultant time is $£ 162$ (including on costs such as administrative support and buildings) and the cost per hour for a non-medical clinician (e.g. CMHT nurse) is $£ 44$. The costs of drugs prescribed by secondary care were derived from the British National Formulary. ${ }^{26}$ The costs of scans were derived on the basis of the Department of Health's Dementia Commissioning Pack. ${ }^{27}$ Costs incurred as a result of time spent on discussion and meetings were based on the size of individual teams, allowing for an average of $6 \mathrm{~min}$ discussion per patient (team sizes and calculations available from the author). The average time taken for domiciliary visit and/or administrative time costs were calculated on the basis of discussions with representatives from professional groups in each of the teams or on data recorded by team members (average times for appointment available from the author on request). The mileage travelled by clinicians was calculated using the Automobile Association Website (www.theaa.com). Travel and transport costs are part of general overheads in the unit costs of health and social care ${ }^{25}$ but as this was anticipated to be an area of difference between the two models, this was calculated separately for each patient seen at the rate of $54 \mathrm{p} / \mathrm{mile}$ up to 3500 miles as suggested in this unit cost document.

\section{Analysis}

Data were analysed using Excel 2007 and Stata Version 12.1 for Windows ( $\chi^{2}$, Fisher's exact tests if less than 5 in a cell, Wilcoxon rank sum test). Parametric and non-parametric tests were applied, as appropriate, to evaluate costs and quality of care provided. To be conservative and because non-parametric distributions were predicted, the cost data were analysed using the Wilcoxon rank sum test.

\section{Results}

\section{Quality data}

A similar range of diagnoses were seen in both areas (Alzheimer's or mixed dementia: 17 in MCS group, 15 in

\begin{tabular}{|c|c|c|c|c|}
\hline & $\begin{array}{c}\text { MCS } \\
\text { group } \\
(n=33)^{\mathrm{a}}\end{array}$ & $\begin{array}{c}\text { CMHT } \\
\text { group } \\
(n=33)^{\mathrm{a}}\end{array}$ & $P$ & Comments \\
\hline Mean age, years & 80 & 84 & 0.03 & Significantly older in Trust B \\
\hline Mean age when leaving school, years & 15 & 15 & $>0.05$ & $\begin{array}{l}\text { Not recorded in } 4 \text { patients in MCS and in } \\
20 \text { patients in CMHT }\end{array}$ \\
\hline Female, $n(\%)$ & $19(58)$ & $22(67)$ & $>0.05$ & \\
\hline MMSE, median & 23.5 & 25 & 0.2 & $n=32$ in both groups \\
\hline ACE-R, median (range) & $67(76)$ & & & $\begin{array}{l}\text { CMHT group not analysed as only } 9 \text { patients } \\
\text { had ACE-R done, } n=31 \text { in MCS group }\end{array}$ \\
\hline $\begin{array}{l}\text { Accommodation - independent/ } \\
\text { sheltered, } n(\%)\end{array}$ & $32(97)$ & $30(94)$ & $>0.05$ & \\
\hline Seen with relative/friend/carer, $n(\%)$ & $33(100)$ & $24(73)$ & $<0.001$ & $\begin{array}{l}\text { Clinicians in CMHT group may have } \\
\text { contacted relative by telephone after interview }\end{array}$ \\
\hline Mean days to be seen (s.e.), $n$ (\%) & $25(3)$ & $20(3)$ & 0.23 & \\
\hline Mean months since symptom onset & 23 & 24 & 0.77 & 12 not known in $\mathrm{CMHT}$ group \\
\hline Received pre-diagnostic counselling, $n(\%)$ & $32(94)$ & $2(6)$ & $<0.0001$ & \\
\hline Dementia blood screen examined, $n(\%)$ & $33(100)$ & $24(73)$ & 0.001 & \\
\hline Physical exam done by GP/psychiatrist, $n(\%)$ & $16(48)$ & $14(42)$ & $\mathrm{n} / \mathrm{s}$ & \\
\hline Functioning examined formally, $n$ (\%) & $24(73)$ & $1(3)$ & $<0.0001$ & e.g. Bristol Activities of Daily Living ${ }^{30}$ \\
\hline Behaviour examined formally, $n(\%)$ & $22(67)$ & $1(3)$ & $<0.0001$ & e.g. Cambridge Behavioural Inventory ${ }^{31}$ \\
\hline Global assessment formal, $n(\%)$ & $33(100)$ & $14(42)$ & $<0.0001$ & e.g. $\mathrm{HoNOS}_{1}^{32}$ EQ-5D-5L ${ }^{33}$ \\
\hline Depression examined formally, $n$ (\%) & $2(6)$ & $1(3)$ & $>0.05$ & e.g. Geriatric Depression Scale ${ }^{34}$ \\
\hline Risk assessment, $n(\%)$ & $31(94)$ & $22(67)$ & 0.02 & \\
\hline Patient/carer sent copy of GP letter, $n(\%)$ & $29(88)$ & $14(42)$ & $<0.0001$ & \\
\hline
\end{tabular}

ACE-R, Addenbrooke's Cognitive Examination-Revised; GP, general practitioner; HoNOS, Health of the Nation Outcome Scales; MMSE, mini-Mental State Examination; $\mathrm{n} / \mathrm{s}$, non-significant

a. Unless otherwise stated. 
CMHT group; vascular dementia: 9 in MCS and 8 in CMHT; Lewy body dementia: 0 in MCS group, 2 in CMHT group; mild cognitive impairment: 6 in both groups; depression: 0 in MCS and 1 in CMHT; other diagnoses: 1 chronic subdural in MCS group and 1 multiple sclerosis-related cognitive impairment in CMHT group). Demographic data and the analysis of data collected during patient assessments are shown in Table 1.

Diagnostic assessments included a clinical assessment of behaviour, functioning and a global assessment of severity in nearly all patients, with no significant differences between groups on these variables. Some structured questionnaires (e.g. EQ-5D, ${ }^{33}$ Cambridge Behavioural Inventory $^{31}$ ) were sent to patients and carers before the actual appointment in the MCS group. Others were administered by clinicians during the clinic appointment. However, structured assessments in patients and carers using symptom rating scales in these domains were not routinely done in the CMHT group. A Mini-Mental State Examination (MMSE) ${ }^{28}$ was performed in all patients except one in each group. In the MCS group, the Addenbrooke's Cognitive Examination-Revised (ACE-R) test ${ }^{29}$ was performed routinely, with a median score of 67 , but it was not performed routinely in the CMHT group. Computed tomography head scans were ordered as part of the assessment to a similar extent in both groups (19 in MCS and 17 in CMHT), whereas scans that had been done previously and considered by the clinician to be recent enough to be helpful amounted to a further 6 in the MCS and 5 in the CMHT group. So, only 8 MCS (24\%) and 11 CMHT (33\%) patients did not have scans available for diagnostic purposes. Scanning is widely available in both trusts and it was patient preference and some clinician guidance that determined whether a patient had a scan or not. Diagnoses were recorded by clinicians in $100 \%$ of letters sent to GPs. A psychologist saw two patients in the MCS group (one for neuropsychological testing and one for cognitive stimulation therapy) and two patients in the CMHT group had further neuropsychological testing.

The post-diagnostic advice given by clinicians to patients and/or carers from the MCS $v$. CMHT group in percentage terms was signposting: to the third sector (70\% v. $24 \%$; $P=0.0002)$; for welfare benefits $(55 \%$ v. $36 \%$; $P>0.05)$; to Social Services (67\% v. 48\%; $P>0.05$ ); advanced planning discussions (55\% v. 45\%; $P>0.05)$. Interviewers were better at documenting whether patients were drivers in the MCS group (information not recorded in three patients in the MCS group and nine patients in the CMHT group). Anti-dementia drugs were prescribed in $65 \%$ of patients eligible for prescriptions (if patients had Alzheimer's disease, mixed dementias or Lewy body dementia and MMSE scores greater than 10). Payment methods differed between groups, with the CMHT passing on prescribing to GPs after 1-2 months compared with the MCS group, where clinicians continued to prescribe for 3-4 months.

\section{Patient pathways}

The major significant difference was that just under half of patients in the CMHT group $(n=15,45 \%)$ were seen only by a doctor at their usual place of residence and then discharged, whereas in the MCS group 97\% were seen by both a doctor and a non-medical clinician (once or twice). In the CMHT group, $55 \%$ of patients were seen by nonmedical clinicians several times (range 2-11). Although some patients received no follow-up in the CMHT group, others received extensive follow-up within the 6-month period, incurring increased travel time, mileage and face-toface costs. Most medical input was from the clinic base for the MCS group, but $85 \%$ of patients also received a domiciliary visit by a non-medical clinician either pre- or post-diagnosis (or both).

\section{Costs of service}

The total costs per person to secondary care between the MCS and the CMHT groups over 6 months were not significantly different in the non-adjusted analysis or the analysis adjusted for age and MMSE score. The mean total cost of care in the MCS group was $£ 742$ (median £722) and in the CMHT group it was $£ 807$ (median $£ 833$ ). Travel costs were significantly higher in the CMHT group where all patients were seen at their usual place of residence $(Z=-5.14, \quad P<0.0001$, effect size -0.63). Consultants travelling to see patients would often add a cost of $£ 162 /$ hour to each assessment in the CMHT group. This expense was not needed for clinic-based assessments by consultants

\begin{tabular}{|lcc|}
\hline $\begin{array}{l}\text { Table } \mathbf{2} \\
\text { Costs }\end{array}$ & Mean costs in memory clinic service $(M C S)$ group and community mental health team (CMHT) group ${ }^{\text {a }}$ \\
\hline Total Costs & MCS group $f($ mean per person \pm s.d.) & CMHT groupf (mean per person \pm s.d.) \\
\hline Direct costs & $272(250)$ & $807(375)$ \\
\hline Office costs & $182(81)$ & $252(124)$ \\
\hline Travel time ${ }^{b}$ & $\mathbf{7 6 ( 5 9 )}$ & $224(130)$ \\
\hline Mileage costs & $28(21)$ & $\mathbf{1 8 6 ( 1 0 6 )}$ \\
\hline Multidisciplinary team costs & $44(15)$ & $32(31)$ \\
\hline Scan costs & $74(67)$ & $30(41)$ \\
\hline Drug costs & $67(103)$ & $69(72)$ \\
\hline
\end{tabular}

Bold denotes significance.

a. Small differences in the total means can be accounted for by rounding off

b. The only significant difference between the groups was in the travel time costs $P<0.0001 ; z=-5.14$ (Wilcoxon rank sum test). The drug, scan and multidisciplinary team costs were not formally analysed as their distributions were not suitable for parametric testing and there were too many ties for the Wilcoxon rank sum test.

\section{Bulletin}


in the MCS group. The costs for non-medical staff to travel to see patients more frequently than in the MCS group also added to this overall travel cost figure. Other costs between groups were not significantly different using non-parametric analysis (Table 2).

\section{Discussion}

This paper highlights the cost and quality differences between two service models for patients referred with concerns about their memory. The total costs to secondary care were less with the MCS-based service than the CMHTbased service (median cost of $£ 722 v$. $£ 833$ per patient), but this difference was not statistically significant. The MCS offered significantly more multidisciplinary care to a greater number of patients than the CMHT service. Both services offered a high-quality diagnostic service but we argue that the MCS service was able to offer more systematic and comprehensive care, including pre-diagnostic counselling, more systematic screening of blood tests for reversible causes of dementia/comorbidity, more extended cognitive examination and structured assessment tools, better evidence of signposting to the third sector as well as copying of letters to patients and carers. It has been demonstrated that there is greater satisfaction with multidisciplinary assessment ${ }^{35,36}$ where diagnostic and management options are explained to both patient and caregiver.

It is possible that patients in the CMHT group were not typical for an MCS or that a selection bias was introduced, with only 33 patients in the CMHT group. However, we feel this is unlikely as the Department of Health memory clinic criteria were applied to all referrals accepted into the study in a systematic way.

We acknowledge that the numbers included in the study were small and the findings can only be regarded as preliminary. However, we cannot exclude the possibility that a CMHT service may be more economical for all types of patients as it was beyond the scope of this study to examine the costs for all patients entering CMHT services in both areas. The study was also not a full economic evaluation where costs and outcome data (such as delays to institutional care) are combined to reach conclusions. The $\mathrm{CSRI}^{23}$ as adapted for this study only examines costs to secondary mental healthcare and not primary care, social care or carer time costs. Using the CSRI, we detailed the patient's involvement with doctors and other clinicians as accurately and comprehensively as possible. In real life, clinicians do not return to base between patient visits so costs may have been inflated in both services for travel time. We were aware that there seemed to be differences between groups in the rate whereby prescribing was handed over to GPs.

This was a retrospective service evaluation and we encountered many of the pitfalls of examining data that were not specifically collected for research purposes. However, the pragmatic design of this study also means it is more reflective of actual practice and therefore less subject to a Hawthorne effect.

Stakeholder views had been sought in both trusts and satisfaction was high with both services in the year of the study, but this was not evaluated specifically in this research and satisfaction cannot be inferred from these data.

There will, of course, be differences among clinicians about what determines the quality of a memory service and we acknowledge our own subjectivity. However, we took a pragmatic view on which variables to include, based on the literature and the information we were likely to be able to obtain from retrospective data in these two services. Other quality indicators for a memory service may be helpful to consider in future studies, for example the rate of reversible causes found, the rate of 'no diagnosis' made, the range of diagnoses or the rate at which drugs were accepted by eligible patients. However, this sample was too small to find significant between-group differences on these indicators.

The IMD in the MCS group was lower than in the CMHT group. This could possibly influence referral patterns but we acknowledge that this is a complex issue, involving the attitudes of patients, families and their referring GPs. Ethnicity was not specifically matched for in this study and this is acknowledged as a study limitation. We acknowledge that both groups had higher than expected rates of patients not receiving anti-dementia drugs. Clinicians did not always offer the drug, because they were concerned patients would not comply with taking the medication. However, some patients refused the drugs because of possible side-effects or other factors.

Another emerging care model in the UK utilises the services of 'allied mental health professionals' in making diagnoses and offering interventions with medical input not provided face to face for most patients. ${ }^{5,37,38}$ It may be argued that some of the diagnostic quality provided by a 'medical' view on diagnosis may be compromised in such services and this needs further evaluation.

\section{Acknowledgements}

We thank all the clinicians who provided information for this project, and Christine Hill (CLAHRC administrator), Sam Norton (statistician for CLAHRC in Public Health Department), David Rubinsztein (statistical support) and $\mathrm{Dr}$ John Battersby (ERPHO) for providing help with demographic and QOF data. J.S.R. thanks her colleagues in psychiatry at the West Suffolk Hospital, who provided support for the project and backfill for sessions during the CLAHRC year of training. J.S.R. also thanks all those in the CLAHRC, Cambridge and Peterborough scheme for their generous support of her fellowship.

\section{About the authors}

Judy Sasha Rubinsztein, Norfolk and Suffolk NHS Foundation Trust, consultant and fellow in NIHR CLAHRC [National Institute for Health Research Collaborations for Leadership in Applied Health research and Care] for Cambridge and Peterborough; Marelna Janse van Rensburg, ST6 in Cambridgeshire and Peterborough NHS Foundation Trust; Zerak Al-Salihy, ST5 in Norfolk and Suffolk NHS Foundation Trust; Deborah Girling, consultant, Cambridgeshire and Peterborough NHS Foundation Trust; Louise Lafortune, senior research associate, NIHR CLAHRC for Cambridgeshire and Peterborough, Cambridge Institute of Public Health, University of Cambridge; Muralikrishnan Radhakrishnan, senior health economist, Centre for the Economics of Mental and Physical Health, Institute of Psychiatry at King's College London; Carol Brayne, Director of the Cambridge Institute of Public Health, Department of Public Health and Primary Care, University of Cambridge. 


\section{References}

1 Department of Health. Living Well with Dementia: A National Dementia Strategy. Department of Health, 2009.

2 Banerjee S, Wittenberg R. Clinical and cost effectiveness of services for early diagnosis and intervention in dementia. Int J Geriatr Psychiatry 2009; 24: 748-54.

3 Wolfs CAG, Dirksen CD, Kessels A, Severens JL, Verhey RJ. Economic evaluation of an integrated diagnostic approach for psychogeriatric patients. Arch Gen Psychiatry 2009; 66: 313-23.

4 Gaugler JE, Kane RL, Kane RA, Newcomer R. Early community-based service utilisation and its effects on institutionalisation in dementia caregiving. Gerontologist 2005; 45: 177-85.

5 Banerjee S, Willlis R, Matthews D, Contell F, Chan J, Murray J. Improving the quality of care for mild to moderate dementia: evaluation of the Croyden Memory Service Model. Int J Geriatr Psychiatry 2007; 22: 782-8.

6 Brodaty H, Gresham M, Luscombe G. The Prince Henry Hospital Dementia Caregivers' Training Programme. Int J Geriatr Psychiatry 1997; 12: 183-92.

7 Mittleman MS, Roth DL, Clay OJ, Haley WE. Preserving health of Alzheimer caregivers: impact of a spouse caregiver intervention. Am J Geriatr Psychiatry 2007; 15: 780-9.

8 Knight BG, Lutzky SM, Macofsky-Urban F. A meta-analytic review of interventions for caregiver distress: recommendations for future research. Gerontologist 1993; 33: 240-8.

9 Logiudice D, Wlatrowicz W, Brown K, Burrows C, Ames D, Flicker L. Do memory clinics improve the quality of life of carers? A randomized pilot trial. Int J Geriatr Psychiatry 1999; 14: 626-32.

10 Luce A, McKeith I, Swann A, Daniel S, O'Brien J. How do memory clinics compare with traditional old age psychiatry services? Int J Geriatr Psychiatry 2001; 16: 837-45.

11 Phipps AJ, O'Brien J. Memory clinics and clinical governance-a UK perspective. Int J Geriatr Psychiatry 2002; 17: 1128-32.

12 Passmore AP, Craig DA. The future of memory clinics. Psychiatr Bull 2004; 28: 375-7.

13 Vernooij-Dassen MJFJ, Moniz-Cook ED, Woods RT, Lepeleire JD, Leuschner $A$, Zanetti $O$, et al. Factors affecting timely recognition and diagnosis of dementia across Europe: from awareness to stigma. Int $J$ Geriatr Psychiatry 2005; 20: 377-86.

14 Kelly C. Memory clinics. Psychiatry 2007; 7: 61-3.

15 Pelosi AJ, McNulty SV, Jackson GA. Role of cholinesterase inhibitors in dementia care needs rethinking. BMJ 2006; 333: 491-3.

16 Coombes R. Evidence lacking for memory clinics to tackle dementia, say critics. BMJ 2009; 338: b55.

17 NHS Employers. Quality and Outcomes Framework guidance for GMS contract 2009/10. NHS Employers, 2009 (http://www. nhsemployers.org/Aboutus/Publications/Documents/QOF_Guidance_ 2009_final.pdf).

18 Department for Communities and Local Government. Indices of Multiple Deprivation. DCLG, 2010 (https://www.gov.uk/government/ publications/english-indices-of-deprivation-2010).

19 Department of Health. Service Specification for Dementia: Memory Service for Early Diagnosis and Intervention (Annex 2, p. 48). Department of Health, 2011
20 National Institute for Health and Care Excellence. Dementia: Supporting People with Dementia and their Carers in Health and Social Care. NICE, 2006.

21 Doncaster E, McGeorge M, Orrell M, Shinkwin L, Browne M. Memory Services National Accreditation Programme (MSNAP): Standards for Memory Services - Assessment and Diagnosis. Royal College of Psychiatrists, 2009.

22 Simpson S, Beavis D, Dyer J, Ball S. Should old age psychiatry develop memory clinics? A comparison with domiciliary work. Psychiatr Bull 2004; 28: 78-82.

23 Beecham J, Knapp M. Costing psychiatric interventions. In Measuring Mental Health Needs (2nd edn) (ed. G Thornicroft): 200-24. Gaskell 2001.

24 National Institute for Health and Care Excellence. Guide to the Methods of Technology Appraisal. NICE, 2013.

25 Curtis L. Unit costs of health and social care. Personal Social Services Research Unit, 2011 (http://www.pssru.ac.uk/project-pages/unit-costs/ 2011/index.php)

26 British Medical Association, Royal Pharmaceutical Society. British National Formulary (September issue). BMJ Books, Pharmaceutical Press, 2011.

27 Department of Health. Dementia Commissioning Pack 2011. Department of Health, 2011.

28 Folstein MF, Folstein SE, McHugh PR. 'Mini-mental state': a practical method for grading the cognitive state of patients for the clinician. $J$ Psychiatr Res 1975; 12: 189-98.

29 Mioshi E, Dawson K, Mitchell J Arnold R, Hodges JR. The Addenbrooke's Cognitive Examination Revised (ACE-R): a brief cognitive test battery for dementia screening. Int J Geriatr Psychiatry 2006; 21: 1078-85.

30 Bucks RS, Ashworth GK, Wilcock GK, Sigfried K. Assessment of activities of daily living in dementia: development of the Bristol Activities of Daily Living Scale. Age Ageing 1996; 25: 113-20.

31 Wear HJ, Wedderburn CJ, Mioshi E, Williams-Gray CH, Mason SL, Barker RA, et al. The Cambridge Behavioural Inventory Revised. Dement Neuropsychol 2008; 2: 102-7.

32 Wing JK, Beevor AS, Curtis RH, Park SB, Hadden S, Burns A. Health of the Nation Outcome Scales (HoNOS). Research and development. Br J Psychiatry 1998; 172: 11-8.

33 EuroQol. EQ-5D. Available at http://www.euroqol.org/eq-5d-products/ eq-5d-5l.html

34 Yesavage JA, Brink TL, Rose $T L$, Lum $O$, Huang $V$, Adey $M B$, et al. Development and validation of a geriatric depression screening scale: a preliminary report. J Psychiatr Res 1983; 17: 37-49.

35 Wolfs CAG, Kessels A, Dirksen CD, Severens JL, Verhey FRJ. Integrated multidisciplinary diagnostic approach for dementia care: randomised controlled trial. Br J Psychiatry 2008; 192: 300-5.

36 Foreman P, Gardener IL, Davis S. Multidisciplinary memory clinics: what is important to caregivers and clients? Int J Geriatr Psychiatry 2004; 29 $588-9$.

37 Page $S$, Hope $K$, Bee $\mathrm{P}$, Burns A Nurses making a diagnosis of dementia - a potential change in practice? Int J Geriatr Psychiatry 2008; 23: 27-33.

38 Page S, Hope K, Maj C, Mathew J, Bee P. 'Doing things differently'working towards distributed responsibility within memory assessment services. Int J Geriatr Psychiatry 2012; 27: 280-5.

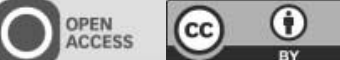

\section{Bulletin}

